\title{
Thermal and energy production optimization in microgrids with Model Predictive Control
}

\author{
S. Raimondi Cominesi ${ }^{1}$, A. Del Corno ${ }^{2}$, E. Corsetti ${ }^{2}$, G. A. Guagliardi ${ }^{2}$, C. Sandroni ${ }^{2}$ and R. Scattolini ${ }^{1}$ \\ ${ }^{1}$ Dipartimento di Elettronica, Informazione e Bioingegneria \\ Politecnico di Milano \\ Via Ponzio 34/5, 20133 Milano (Italy) \\ Phone number: +39 02 23994028, e-mail: stefano.raimondi@polimi.it, riccardo.scattolini@polimi.it
}

${ }^{2}$ Ricerca per il Sistema Energetico italiano, RSE S.p.A.

www.rse-web.it

Phone number: +39 02 39921, e-mail: ada.delcorno@ @rse-web.it, edoardo.corsetti@ rse-web.it, giuseppe.guagliardi@rse-web.it, carlo.sandroni@ rse-web.it

\begin{abstract}
This paper presents an algorithm for the management of electrical and thermal power production in microgrids connected to the utility grid and subject to uncertainties in both load requests and non-dispatchable generation.

The suggested procedure allows, on one hand, to obtain the dayahead setpoint profiles for the microgrid devices, resulting in the economic optimal power exchange with the grid. On the other hand, it supplies a suitable method to refine such setpoint profiles, during the daily execution, in order to cope with unpredicted behaviours of the system. A formulation of the algorithm for a possible case study is given and simulation results are illustrated in the final part of the work.
\end{abstract}

\section{Key words}

optimal energy management, distributed generation, combined cooling heat and power system, uncertainty

\section{Introduction}

The importance of energy management in micro-grids is connected to the ever increasing diffusion of distributed generation in the electric energy production systems (see e.g. [1], [2]) and testified by the continuously growing interest of research on such topic (e.g. [3], [4] and references therein). These considerations are all the more true when thermal power generation is taken into account as well. The advantages deriving from an overall optimal management policy in microgrids are, infact, easily imaginable and can range from simple economic savings for the owners, to a global $\mathrm{CO}_{2}$ emission reduction.

Although some work has already been done on this argument (e.g. the same [4]), the problem is still an open topic.

In this paper, we present an algorithm that, considering an estimated power request profile, together with nondispatchable production prediction and temperature forecast, is capable to produce a day-ahead optimal energy

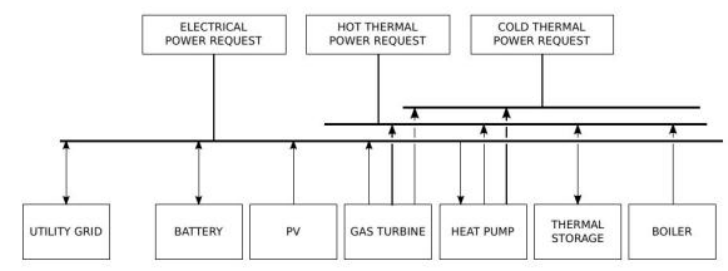

Fig. 1. Contributes of the MG devices

exchange with the network and the corresponding setpoints for the microgrid devices. During the daily production plan execution, possible errors in the forecasted input data represent a threat to the ability to comply with the computed optimal exchange profile with the utility grid. A method capable to modify the production plan, in order to cope with such errors is therefore introduced. Finally a simulation of a proposed case study is shown in order to illustrate the possible results of this approach. Although some of the device models introduced in the next session may appear specific, the proposed approach is, by any means, general and adaptable to similar cases. Preliminary results concerning this research have been presented in [5], where however only the electrical energy production was considered, while the management of the thermal aspects was neglected.

The rest of the paper is organized as follows: Section 2 contains the model description of the microgrid used as an example to present the algorithm. The optimization problems are then formalized in Section 3 while the simulation results are shown in Section 4. Finally some conclusions are drawn in Section 5.

\section{Description of the system}

The case study microgrid (MG) considered in this work comprises multiple devices that need to be efficiently coordinated in order to satisfy the electrical power request 
$y_{\mathrm{L}}{ }^{\text {elt }}$ and both the hot $y_{\mathrm{L}}^{\mathrm{h}}$ and cold $y_{\mathrm{L}}^{\mathrm{c}}$ thermal power request. In particular, as shown in the scheme in Figure 1, the microgrid is equipped with a dispatchable generator (i.e. a gas turbine, denoted with the letter T), an electrical storage device (i.e. a battery, denoted with ES) and a nondispatchable generator (i.e. photovoltaic panels identified by the letter $\mathrm{P}$ ) that, together with the power drained from the distribution network (hereafter denoted with the letter $\mathrm{N}$ ), contribute to balance the electrical power request. According to the chosen machine setup, the gas turbine can be used also to help coping with either the hot or the cold thermal power request. The same consideration holds true also for the heat pump (identified by the letter $\mathrm{H}$ ) that acts as a converter from the electrical power to the thermal ones. To complete the list of the devices, a boiler (B) and a thermal storage unit (TS) contributing to satisfy the hot thermal power request are considered.

The dispatchable units of the MG are supposed to be endowed with embedded controllers, so that the dynamics of the devices that concerns the modeling phase are constituted by the I/O relationship between the desired setpoints, control variables of the system, and the actual power output.

The photovoltaic panels are supposed not to be adjustable, so that the totality of its produced power $y_{P}$ flows through the MG. The electrical loads are not controllable as well and are considered to be mandatorily satisfied. Daily predictions of both the PV power production and power requests are assumed to be available.

The connection to $\mathrm{N}$ is bidirectional and allows for both purchase and sale of electric power. The net power exchanged between the MG and $\mathrm{N}$ (positive if power is absorbed) is denoted by $y_{\mathrm{N}}$. The purchase cost and the sale price are known but not constant (subject to market fluctuations)

For the definition of the discrete-time models of the MG components we adopted the sampling period $\tau=15$ minutes.

\section{A. Model of the electrical storage system}

The storage device has been essentially modeled as an integrator with internal state $x_{\mathrm{ES}}$ representing its normalized state of charge (SOC). Since the charge efficiency $\eta_{\mathrm{CH}}$ is generally different from the discharge efficiency $\eta_{\mathrm{DCH}}$, we considered two input for the model: $u_{\mathrm{ES}}{ }^{\mathrm{CH}}(\mathrm{k})$ and $u_{\mathrm{ES}}{ }^{\mathrm{DCH}}(\mathrm{k})$, representing the absolute value of the power absorbed and released, respectively, from the battery. In so doing, the SOC dynamics is described by the following system:

$\left\{\begin{array}{l}x_{\mathrm{ES}}(k+1)=x_{\mathrm{ES}}(k)+\frac{\tau}{C_{\mathrm{ES}}^{\max }}\left(\eta_{\mathrm{CH}} u_{\mathrm{ES}}^{\mathrm{CH}}(k)-\eta_{\mathrm{DCH}} u_{\mathrm{ES}}^{\mathrm{DCH}}(k)\right) \\ y_{\mathrm{ES}}(k)=u_{\mathrm{ES}}^{\mathrm{DCH}}(k)-u_{\mathrm{ES}}^{\mathrm{CH}}(k),\end{array}\right.$

where $C_{\mathrm{ES}}{ }^{\mathrm{max}}$ is the nominal capacity of the storage system. We considered a battery with $C_{\mathrm{ES}}{ }^{\mathrm{max}}=32 \mathrm{kWh}$. The output of the system $y_{\mathrm{ES}}(\mathrm{k})$ represents the power exchange between the battery and the microgrid, intended positive if flowing out from the storage device.

To complete the description, the following operational constraint is included:

$$
u_{\mathrm{ES}}^{\mathrm{CH}}(k) \cdot u_{\mathrm{ES}}^{\mathrm{DCH}}(k)=0, \quad \forall k \in \mathbb{N} .
$$

Notice that, under condition (2), model (1) is equivalent to a piecewise linear system. The charge and discharge power setpoints are upper bounded as well, so that, $\forall \mathrm{k} \in \mathrm{N}$, the constraints

$$
\left\{\begin{array}{l}
0 \leq u_{\mathrm{ES}}^{\mathrm{CH}}(k) \leq u_{\mathrm{ES}}^{\max } \\
0 \leq u_{\mathrm{ES}}^{\mathrm{DCH}}(k) \leq u_{\mathrm{ES}}^{\max }
\end{array}\right.
$$

are enforced. For the ES we used, $u_{\mathrm{ES}}{ }^{\max }=30 \mathrm{~kW}$. Finally, in order to reduce the risk of damaging the storage unit, the state of charge is bounded between $x_{\mathrm{ES}}{ }^{\min }(\mathrm{k})=0.1$ and $x_{\mathrm{ES}}{ }^{\max }(\mathrm{k})=0.9$.

\section{B. Model of the turbine}

The model of the gas turbine is derived from the device available at the RSE test facility in Milan. This turbine has an embedded controller based on a four states automata, namely: starting, running, stopping and stopped.

When the system is in the running mode, experimental tests carried out on the gas turbine allowed us to identify the relationship between machine set point $u_{\mathrm{T}}(\mathrm{k})$ and actual output $y_{\mathrm{T}}^{\mathrm{elt}}(\mathrm{k})$ in terms of the following transfer function:

$$
G_{\mathrm{T}}(s)=\frac{(1+11.25 s)}{(1+6.41 s)(1+1.36 s)} e^{-26.4 s} .
$$

As reasonably expected, the settling time of such system (approximately 1 minute) motivates the adoption of a zero order model. In other words, in running mode,

$$
y_{\mathrm{T}}^{\text {elt }}(k)=u_{\mathrm{T}}(k) \text {. }
$$

The setpoint $u_{\mathrm{T}}$ and, consequently, the corresponding steady-state power output of T, $y_{\mathrm{T}}{ }^{\text {elt }}$, are bounded between the values $u_{\mathrm{T}}^{\text {min }}=50 \mathrm{~kW}$ and $u_{\mathrm{T}}^{\text {min }}=100 \mathrm{~kW}$.

Taking the consumption of the auxiliary actuators into account (i.e., the fuel pump and the internal cooling system), the net contribution of the gas turbine to the microgrid electrical power balance is negligible during the transient phases constituted either by the starting or by the stopping state. The stopping state lasts an amount of time consistent with $\tau$, while the duration of the starting phase changes according to the current machine's status. In particular, depending on whether it is cold or hot, the time required for the start-up (latency time) takes value $t_{\text {cold }}$ or $t_{\text {hot }}$, respectively. Let $t$ be the time taken by $\mathrm{T}$ to become cold. Furthermore, to avoid damages and improper use of the device, the turbine must be in operation, once started, at least for a given minimum operation time $t_{\text {delay. }}$.

From the electrical point of view, the input of the model are the boolean on/off signal $\delta_{\mathrm{T}}{ }^{\text {elt }}(\mathrm{k})$ and the power setpoint $u_{\mathrm{T}}(\mathrm{k})$. In order to model the logical states of $\mathrm{T}$, two integer variables, namely $t_{\mathrm{on}}(\mathrm{k})$, and $t_{\mathrm{off}}(\mathrm{k})$, are introduced. In particular, variable $t_{\text {on }}$ denotes the number of further time steps required to complete the latency time from the start signal, while $t_{\text {off }}$ denotes the time steps spent since the last switch off. Consistently with (4), when $\mathrm{T}$ is on (i.e., if $\delta_{\mathrm{T}}{ }^{\text {elt }}(\mathrm{k})=1$ and $\left.t_{\mathrm{on}}(\mathrm{k}) \leq 0\right)$, then $y_{\mathrm{T}}{ }^{\text {elt }}(\mathrm{k})=u \mathrm{~T}^{\text {elt }}(\mathrm{k}) ; \quad$ on the contrary, if $\delta_{\mathrm{T}}^{\mathrm{elt}}(\mathrm{k})=0$ (i.e., no switch-on signal has been delivered) or $t_{\mathrm{on}}(\mathrm{k})>0$ (i.e., $\mathrm{T}$ is in the starting phase), then $y_{\mathrm{T}}^{\text {elt }}(\mathrm{k})=0$.

At each time step, the auxiliary variables are updated as follows:

$$
t_{\mathrm{on}}(k+1)= \begin{cases}t_{\mathrm{on}}(k)-1 & \text { if } \delta_{\mathrm{T}}^{\text {elt }}(k)=1 \\ t_{\mathrm{hot}} & \text { if }\left(\delta_{\mathrm{T}}^{\text {elt }}(k)=0\right) \wedge\left(t_{\mathrm{off}}(k)<t\right) \\ t_{\mathrm{cold}} & \text { if }\left(\delta_{\mathrm{T}}^{\text {elt }}(k)=0\right) \wedge\left(t_{\mathrm{off}}(k) \geq t\right),\end{cases}
$$




$$
t_{\text {off }}(k+1)= \begin{cases}t_{\text {off }}(k)+1 & \text { if } \delta_{\mathrm{T}}^{\text {elt }}(k)=0 \\ 0 & \text { if } \delta_{\mathrm{T}}^{\text {elt }}(k)=1 .\end{cases}
$$

Finally, if $-t_{\text {delay }} \leq t_{\mathrm{on}}(\mathrm{k}) \leq 0$, then $\delta_{\mathrm{T}}^{\text {elt }}(\mathrm{k})=1$.

The gas turbine can also be used to produce either heating $\left(y_{\mathrm{T}}^{\mathrm{h}}\right)$ or cooling $\left(y_{\mathrm{T}}^{\mathrm{c}}\right)$ thermal power. In both cases, the production can be expressed as an affine function of the electrical power output. Consistently with these properties the following equations are introduced in the model:

$$
\left\{\begin{array}{l}
y_{\mathrm{T}}^{\mathrm{h}}(k)=\delta_{\mathrm{T}}^{\mathrm{t}}(k)\left(\alpha^{\mathrm{h}} y_{\mathrm{T}}^{\text {elt }}(k)+\beta^{\mathrm{h}}\right) \\
y_{\mathrm{T}}^{\mathrm{c}}(k)=\left(1-\delta_{\mathrm{T}}^{\mathrm{t}}(k)\right)\left(\alpha^{\mathrm{c}} y_{\mathrm{T}}^{\text {elt }}(k)+\beta^{\mathrm{c}}\right),
\end{array}\right.
$$

where $\alpha$ and $\beta$ are the affine function parameters and $\delta \mathrm{T}^{\mathrm{t}}$ is an additional boolean input variable defining the thermal production mode.

\section{Model of the boiler}

For the purpose of this work, with boiler it is intended a generical dispatchable hot thermal power generator. Considering the sampling period adopted for the model, we reasonably assume that the device is constantly in steady state and choose, therefore, the following algebraic equation to represent the dynamic of such generator:

$$
y_{\mathrm{B}}(k)=u_{\mathrm{B}}(k) \text {. }
$$

We consider as operational bounds $u_{\mathrm{B}}{ }^{\min }=0 \mathrm{kWt}$ and $u_{\mathrm{B}}{ }^{\max }$ $=150 \mathrm{kWt}$, with

$$
u_{\mathrm{B}}^{\min } \leq u_{\mathrm{B}}(k) \leq u_{\mathrm{B}}^{\max } .
$$

\section{Model of the heat pump}

The electrical heat pump is a device absorbing electrical power in order to produce either heating or cooling thermal power. In heating mode, the heat pump drains heat from the outside environment and conveys it inside the building. The transfered heat over the absorbed electrical energy ratio, known as COP (Coefficient Of Performance) is the standard parameter used by manufacturers to define the process efficiency. In cooling mode, heat flow direction is reversed: in this case the process efficiency is commonly represented by the absorbed heat over absorbed electrical energy ratio, known as EER (Energy Efficiency Ratio). The values assumed by both the COP and EER parameters are strictly dependant by the conditions under which the machine operates. Consistently with [6], we focused our attention on the dependence from the environmental temperature. In particular, assuming temperature forecasts available, we considered the time-varying efficiency parameters $\operatorname{COP}(\mathrm{T})$ and $\operatorname{EER}(\mathrm{T})$, obtained through identified non-linear mapping functions.

The model we used for taking into account the contribution of the heat pump to the MG has two input variables: $u_{\mathrm{H}}$, the absolute value of the power request and $\delta_{\mathrm{H}}$, a boolean variable expliciting the operating mode of the heat pump. In particular, $\delta_{\mathrm{H}}=0$ if the device is working in cooling mode and $\delta_{\mathrm{H}}=1$ in the opposite case. The outputs of the model are the absorbed electrical power $y_{\mathrm{H}}{ }^{\mathrm{elt}}$, and the two thermal power output: $y_{\mathrm{H}}^{\mathrm{h}}, y_{\mathrm{H}}{ }^{\mathrm{c}}$ (where the use of the superscript

\footnotetext{
${ }^{1}$ where it is intended $u_{\mathrm{ES}}(\mathrm{k})=\left[u_{\mathrm{ES}}{ }^{\mathrm{CH}}(\mathrm{k}) u_{\mathrm{ES}}{ }^{\mathrm{DCH}}(\mathrm{k})\right]^{\mathrm{T}}$ and $u_{\mathrm{TS}}(\mathrm{k})=\left[u_{\mathrm{TS}}{ }^{\mathrm{IN}}(\mathrm{k}) u_{\mathrm{TS}}{ }^{\mathrm{OUT}}(\mathrm{k})\right]^{\mathrm{T}}$
}

letter is analogous to the one used in the turbine model). Consistently with this, the heat pump is defined by the following equations:

$$
\left\{\begin{array}{l}
y_{\mathrm{H}}^{\mathrm{h}}(k)=\delta_{\mathrm{H}} u_{\mathrm{H}}(k) \\
y_{\mathrm{H}}^{\mathrm{c}}(k)=\left(1-\delta_{\mathrm{H}}\right) u_{\mathrm{H}}(k) \\
y_{\mathrm{H}}^{\text {elt }}(k)=\frac{y_{\mathrm{H}}^{\mathrm{h}}(k)}{\operatorname{COP}(T)}+\frac{y_{\mathrm{H}}^{\mathrm{c}}(k)}{\operatorname{EER(T)}}
\end{array}\right.
$$

The output variables of the heat pump are bounded by the values $y_{\mathrm{H}}{ }^{\mathrm{min}}=0 \mathrm{kWt}$ and $y_{\mathrm{H}}{ }^{\max }=80 \mathrm{kWt}$, so that it holds

$$
\begin{aligned}
& y_{\mathrm{H}}^{\min } \leq y_{\mathrm{H}}^{\mathrm{h}} \leq y_{\mathrm{H}}^{\max }, \\
& y_{\mathrm{H}}^{\min } \leq y_{\mathrm{H}}^{\mathrm{c}} \leq y_{\mathrm{H}}^{\max } .
\end{aligned}
$$

\section{Model of the thermal storage}

The model of the thermal storage device is similar to the electrical one. We considered a system constituted by a 5000 liters water tank exchanging thermal power with asymmetric efficiency coefficients $\theta_{\mathrm{IN}}$ and $\theta_{\text {out. The }}$ temperature of the system can span between $70^{\circ} \mathrm{C}$ and $80^{\circ}$ $\mathrm{C}$, its value, normalized, is represented by the state variable $x_{\mathrm{TS}}$. Similarly to the system in (1), splitting the input in two different input variables according to the direction of the thermal power flow $\left(u_{\mathrm{TS}}{ }^{\mathrm{OUT}}\right.$ and $\left.u_{\mathrm{TS}}{ }^{\mathrm{IN}}\right)$, we define the model:

$$
\left\{\begin{aligned}
x_{\mathrm{TS}}(k+1)= & (1-\lambda) x_{\mathrm{TS}}(k)+\frac{\tau}{C_{\mathrm{TS}}}\left(\theta_{\mathrm{IN}} u_{\mathrm{TS}}^{\mathrm{IN}}(k)+\right. \\
& \left.-\theta_{\mathrm{OUT}} u_{\mathrm{TS}}^{\text {OUT }}(k)\right) \\
y_{\mathrm{TS}}(k)= & u_{\mathrm{TS}}^{\text {OUT }}(k)-u_{\mathrm{TS}}^{\mathrm{IN}}(k)
\end{aligned}\right.
$$

where $C_{\mathrm{TS}}$ represents the heat capacity of the water tank and $\lambda$ stands for the percentage of thermal energy lost at every time-step. Finally, assuming constant values for the temperature of the heating source $\left(T_{\mathrm{H}}\right)$ and for the temperature at which the heat is drained $\left(T_{\mathrm{D}}\right)$, the following constraints are written in order to take into account the limitation of the thermal power exchange imposed by the maximum mass flow rate of the storage pump $\left(\rho_{\max }\right)$ :

$$
u_{\mathrm{TS}}(k) \leq \rho_{\max } c_{\mathrm{TS}} \Delta T\left(x_{T S}, T_{H}\right)
$$

\section{E. The overall model of the microgrid}

The models of the devices presented above are finally combined together and the overall microgrid model is defined by the use of the mixed logical dynamical (MLD) system [7] formalism. Such reformulation is based on the definition of a set of auxiliary variables (either continuous or binary) included in a vector $w_{\text {MLD }}$, which allow for converting all the previously introduced logic relations to a set of linear inequalities with binary variables. The process can be completed resorting to the freely available software tool HYSDEL [8]. Thus, letting

$$
\begin{gathered}
x_{\mathrm{MLD}}(k)=\left[x_{\mathrm{ES}}(k) t_{\mathrm{on}}(k) t_{\mathrm{off}}(k) x_{\mathrm{TS}}(k)\right]^{\mathrm{T}}, \quad \text { and } \\
u_{\mathrm{MLD}}(k)=\left[u_{\mathrm{ES}}(k) \delta_{\mathrm{T}}^{\mathrm{t}}(k) u_{\mathrm{T}}(k) \delta_{\mathrm{T}}^{\text {elt }}(k) u_{\mathrm{B}}(k) \delta_{\mathrm{H}}(k) u_{\mathrm{H}}(k) u_{\mathrm{TS}}(k)\right]^{\mathrm{T}}
\end{gathered}
$$

be the state and input vectors ${ }^{1}$ respectively, the overall MLD model takes the form 
$\begin{cases}x_{\mathrm{MLD}}(k+1) & =A x_{\mathrm{MLD}}(k)+B_{\mathrm{u}} u_{\mathrm{MLD}}(k)+B_{\mathrm{aux}} w_{\mathrm{MLD}}(k) \\ y_{\mathrm{MLD}}(k) & =C x_{\mathrm{MLD}}(k)+D_{\mathrm{u}} u_{\mathrm{MLD}}(k)+D_{\mathrm{aux}} w_{\mathrm{MLD}}(k) \\ E_{\mathrm{aff}} & \geq E_{\mathrm{x}} x_{\mathrm{MLD}}(k)+E_{\mathrm{u}} u_{\mathrm{MLD}}(k)+E_{\mathrm{aux}} w_{\mathrm{MLD}}(k)\end{cases}$

Notice that the output $y_{\text {MLD }}$ of the system is a vector composed as follows:

$$
y_{\mathrm{MLD}}(k)=\left[\begin{array}{c}
y_{\mathrm{MLD}}^{\mathrm{elt}}(k) \\
y_{\mathrm{MLD}}^{\mathrm{h}}(k) \\
y_{\mathrm{MLD}}^{\mathrm{c}}(k)
\end{array}\right],
$$

with obvious meaning of the symbology.

As already discussed, the system has to satisfy altogether three different load requests. From the electrical point of view, due to the connection with the distribution network, the following constraints hold:

$$
\begin{gathered}
y_{\mathrm{MLD}}^{\mathrm{elt}}(k)+y_{\mathrm{P}}(k)+y_{\mathrm{N}}(k)=y_{\mathrm{L}}(k), \quad \forall k=0, \ldots, 95 \\
\left\{\begin{array}{l}
y_{\mathrm{MLD}}^{\mathrm{h}}(k) \geq y_{\mathrm{L}}^{\mathrm{h}}(k) \\
y_{\mathrm{MLD}}^{\mathrm{c}}(k) \geq y_{\mathrm{L}}^{\mathrm{c}}(k)
\end{array}\right.
\end{gathered}
$$

\section{The optimization problem}

The aim of the algorithm is to optimize the cost of the electric and thermal production during the day, while satisfying the three nominal load request profiles, and in accordance with the dynamics and the operational constraints on the devices.

Taking into account the non-dispatchable production forecast and the loads' predictions, the algorithm computes the initial operation plan of each MG element for the whole day ahead. The initial planned energy exchanges with the utility grid, corresponding to the network power profile denoted as

$$
y_{\mathrm{N}}^{\mathrm{ref}}(k), \quad k=0,1, \ldots, 95,
$$

is shared with the distribution network manager and acts as a reference plan during the daily execution. In case of unexpected behaviours of the estimated variables, or in presence of more reliable forecasts, the system is able to provide an update to the devices production profiles. Although a new plan can be devised, the resulting component $y_{\mathrm{N}}$ should be as close as possible to the reference plan, in order to avoid penalties from the network manager. As illustrated in Subsection 3-B, this requirement necessarily modifies the cost function resulting in the definition of a different optimization problem.

\section{A. Initial optimization problem}

The cost function to be minimized accounts for:

- the operational cost of the turbine (denoted by $c_{\mathrm{T}}$ ) and its start-up cost $\left(c_{\mathrm{T}}{ }^{\mathrm{st}}\right)$

- the trade prices with the network: the cost of the energy absorbed $\left(c_{\mathrm{N}}{ }^{\mathrm{a}}\right)$ and the price of the energy sold $\left(c_{\mathrm{N}}^{\mathrm{s}}\right)$

- the operational cost of the boiler (denoted by $c_{\mathrm{B}}$ )

Finally, a term $\left(c_{\mathrm{ES}}^{\mathrm{v}}\right)$ penalizing the entity of the battery power setpoint variation is included in order to preserve its life-expectancy. The objective function is hence defined by:

$$
\begin{aligned}
J^{\mathrm{ref}}=\sum_{k=0}^{95} & c_{\mathrm{T}}(k) y_{\mathrm{T}}(k)+c_{\mathrm{T}}^{\mathrm{st}}(k) \max \left\{\delta_{\mathrm{T}}(k)-\delta_{\mathrm{T}}(k-1), 0\right\}+ \\
& +c_{\mathrm{N}}^{\mathrm{a}}(k) \max \left\{y_{\mathrm{N}}(k), 0\right\}+c_{\mathrm{N}}^{\mathrm{s}}(k) \min \left\{y_{\mathrm{N}}(k), 0\right\}+ \\
& +c_{\mathrm{B}}(k) y_{\mathrm{B}}(k)+c_{\mathrm{ES}}^{\mathrm{v}}(k)\left|y_{\mathrm{ES}}(k)-y_{\mathrm{ES}}(k-1)\right|,
\end{aligned}
$$

and the optimization problem to be solved is

$$
\min _{u_{\mathrm{MLD}}(0: 95)} J^{\mathrm{ref}}
$$

where $u_{\mathrm{MLD}}(0: 95)$ stands for the sequence $u_{\mathrm{MLD}}(0), \ldots$, $u_{\mathrm{MLD}}(95)$, subject to: the dynamics (12), the load satisfaction constraints (13), (14) and the operational constraints of the devices. In detail, for all $\mathrm{k}=0, \ldots, 95, u_{\mathrm{ES}}{ }^{\mathrm{CH}}(\mathrm{k}) \in\left[0, u_{\mathrm{CH}}{ }^{\max }\right]$, $u_{\mathrm{ES}}{ }^{\mathrm{DCH}}(\mathrm{k}) \in\left[0, u_{\mathrm{DCH}}{ }^{\max }\right], \quad x_{\mathrm{ES}}(\mathrm{k}) \in[0.1,0.9], \quad u_{\mathrm{ES}}{ }^{\mathrm{CH}}(\mathrm{k})=0 \quad \mathrm{~V}$ $u_{\mathrm{ES}}{ }^{\mathrm{DCH}}(\mathrm{k})=0, \quad u_{\mathrm{T}}(\mathrm{k}) \in\left[u_{\mathrm{T}}{ }^{\min }, \quad u_{\mathrm{T}}{ }^{\max }\right], \quad u_{\mathrm{B}}(\mathrm{k}) \in\left[0, \quad u_{\mathrm{B}}{ }^{\max }\right]$, $u_{\mathrm{H}}{ }^{\mathrm{h}}(\mathrm{k}) \in\left[0, u_{\mathrm{H}}^{\mathrm{h}, \max }\right], u_{\mathrm{H}}{ }^{\mathrm{c}}(\mathrm{k}) \in\left[0, u_{\mathrm{H}}{ }^{\mathrm{c}, \max }\right], u_{\mathrm{TS}}{ }^{\mathrm{IN}}(\mathrm{k}) \in\left[0, u_{\mathrm{IN}}{ }^{\max }\right]$, $u_{\mathrm{TS}}{ }^{\mathrm{OUT}}(\mathrm{k}) \in\left[0, u_{\mathrm{OUT}}{ }^{\mathrm{max}}\right], x_{\mathrm{TS}}(\mathrm{k}) \in[70,80]$ and $u_{\mathrm{TS}}{ }^{\mathrm{IN}}(\mathrm{k})=0 \mathrm{~V}$ $u_{\mathrm{TS}}{ }^{\mathrm{OUT}}(\mathrm{k})$. Problem (16) is a mixed-integer linear program (MILP), which can be readily solved by available software tools.

\section{B. Update of the plan}

As it will be further illustrated in Section 4, whenever the input forecast of the algorithm result to be unexpectedly wrong or, in general, when more reliable forecasts are available, a wise refinement of the previously computed setpoint profiles for the microgrid devices may allow to better compensate for the initial forecast error, while trying to limit as much as possible the discrepancies with $y_{\mathrm{N}}{ }^{\mathrm{ref}}(\mathrm{k})$. In order to accomplish this task, a new optimization problem has to be implemented. The cost function associated to such updating optimization problem is defined by

$$
\begin{aligned}
J^{\mathrm{upd}}=\sum_{k=\bar{k}}^{95} & c_{\mathrm{T}}(k) y_{\mathrm{T}}(k)+c_{\mathrm{T}}^{\mathrm{st}}(k) \max \left\{\delta_{\mathrm{T}}(k)-\delta_{\mathrm{T}}(k-1), 0\right\}+ \\
& +c_{\mathrm{N}}(k)\left|y_{\mathrm{N}}^{\mathrm{ref}}(k)-y_{\mathrm{N}}(k)\right|+c_{\mathrm{B}}(k) y_{\mathrm{B}}(k)+ \\
& +c_{\mathrm{ES}}^{\mathrm{v}}(k)\left|y_{\mathrm{ES}}(k)-y_{\mathrm{ES}}(k-1)\right|,
\end{aligned}
$$

where the terms appearing in (15) and quantifying the cost/price for the energy exchanged with the network are replaced by a term penalizing the discrepancy from the reference plan. The resulting optimization problem is therefore,

$$
\min _{u_{\mathrm{MLD}}(\bar{k}: 95)} J^{\mathrm{upd}},
$$

subject to: the dynamics (12), the load balances (13), (14), and the same operational constraints of the devices as in problem (16).

\section{Simulation study}

Regarding the simulation test, we considered the input data collected in Figure 2. In particular, the solid lines in (a)-(c) illustrate, respectively, the prediction for the electrical power load, together with the expected hot and cold thermal power requests. These are the requests to be mandatorily satisfied by the microgrid. The forecasts of photovoltaic panels production and environmental temperature are shown by the solid lines in (d) and in (e). Finally, (f) collects the unitary trade energy prices and the production 


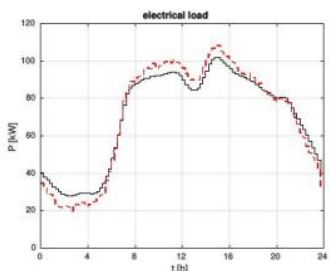

(a)

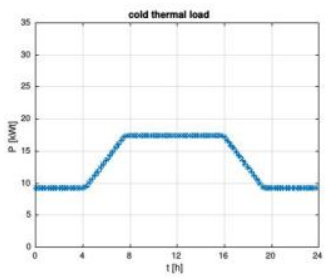

(c)

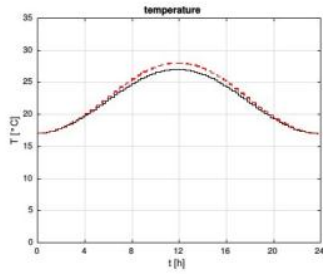

(e)

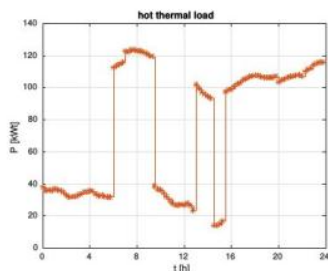

(b)

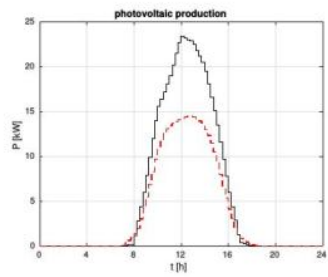

(d)

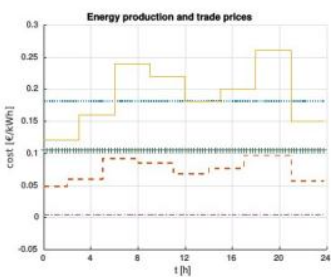

(f)
Fig. 2. Input data: (a) Electrical load, forecasted and real values (solid and dashed line respectively); (b) hot thermal power request; (c) cold thermal power request, (d) photovoltaic production, forecasted and real values (solid and dashed line respectively); (e) external temperature, forecasted and real values (solid and dashed line respectively); (f) Production and trade energy prices: $c_{\mathrm{T}}$ (blue dotted line), $c_{\mathrm{N}}^{\mathrm{a}}$ (yellow solid line), $c_{\mathrm{N}}^{\mathrm{s}}$ (red dashed line), $c_{\mathrm{B}}$ (green plus-marked line), $c_{\mathrm{ES}}^{\mathrm{V}}$ (purple dash-dotted line)

costs. The start-up cost for the gas turbine is constant and equal to $1.49 €$.

According to the day-ahead forecasted input data and the trade energy prices, the algorithm allows to define the optimal daily production plan. Figure 3 shows how the electric power production is distributed among the different devices composing the microgrid. The optimal power exchange profile is represented by the dotted red line while the other curves define the devices' setpoint that whould allow to comply to such profile. Figure 3 shows how the electric power production is distributed among the different devices composing the microgrid. The optimal power exchange profile is represented by the dotted red line while the other curves define the devices' setpoint that whould allow to comply to such profile. In Figure 4 are instead reported the thermal power production profiles of the components that should be adopted in order to safisfy the predicted requests.

It is clear that any open-loop implementation of this production plan is subject to possible errors, due to the uncertain knowledge of the forecasted input values. In order to partially cope with this issue, whenever corrections of the forecasted profiles may be accessible during the daily execution of the plan, it is possible to resort to the update policy presented in Subsection 3-B. Considering for example that the actual electrical load consumption, the photovoltaic production, and the outside temperature are more consistent with the dashed lines than with the solid lines in Figure 2 (a), (d), and (e) respectively, the initial plan would be bound to commit an error of approximately $144 \mathrm{kWh}$ in regard to the energy exchange

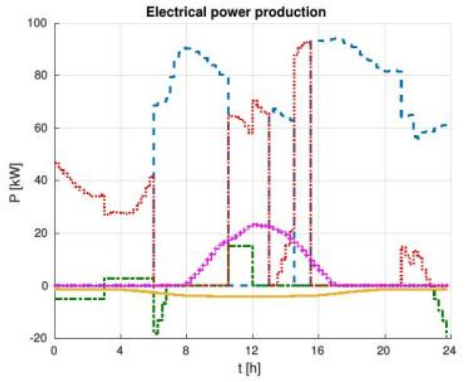

Fig. 3. Electrical power production plan: $y_{\mathrm{T}}^{\text {elt }}$ (dashed blue line), $y_{\mathrm{N}}$ (dotted red line), $y_{\mathrm{ES}}$ (green dash-dot line), $y_{\mathrm{H}}^{\mathrm{c}}$ (yellow solid line), $y_{\mathrm{P}}$ (magenta plus-marked line)

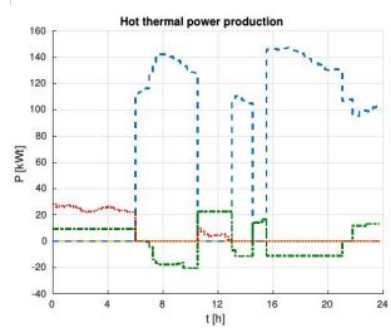

(a)

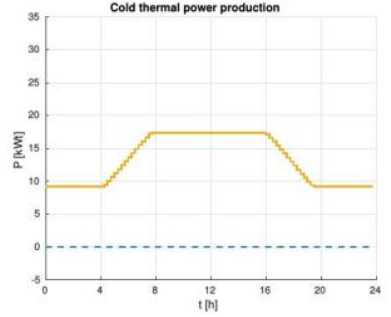

(b)
Fig. 4. (a) Hot thermal production plan: $y_{\mathrm{T}}^{\mathrm{h}}$ (dashed blue line), $y_{\mathrm{B}}$ (dotted red line), $y_{\mathrm{TS}}$ (green dash-dot line), $y_{\mathrm{H}}^{\mathrm{c}}$ (yellow solid line); (b) Cold thermal production plan: $y_{\mathrm{T}}^{\mathrm{c}}$ (dashed blue line), $y_{\mathrm{H}}^{\mathrm{c}}$ (yellow solid line).

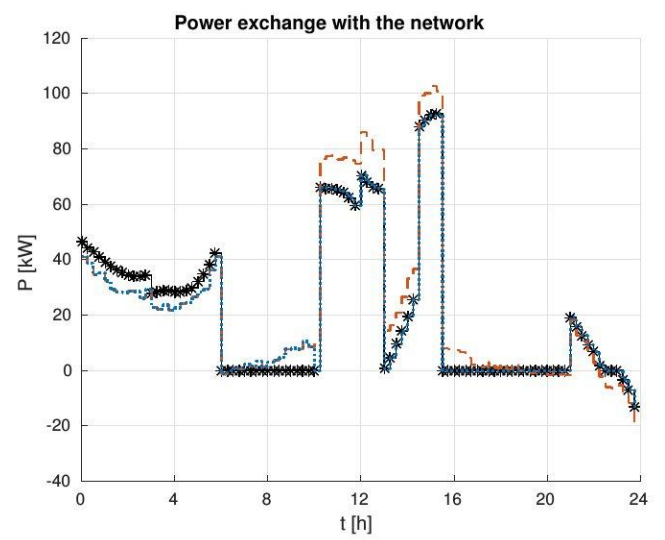

Fig. 5. Effect of the update policy on the power exchange with the network: agreed $y_{\mathrm{N}}^{\text {ref }}$ (star-marked black line) and $y_{\mathrm{N}}$ with and without update of the input variables (dotted blue line and dashed red line respectively)

with the network. Such error might though be corrected revising the optimization plan in accordance to the more reliable data. Figure 5 shows the profiles of the power exchange in the different cases. The dashed red line, representing the power actually exchanged with the network without any correction of the initial plan, drifts away from the agreed profile $y_{\mathrm{N}}{ }^{\text {ref }}$. Assuming the more reliable forecasts to be available around 10 a.m., instead, the production plan can be revised accordingly in order to compensate for the initially erroneous inputs (as the dotted blue line shows).

It should be noted that, even not considering any variation of the thermal request in the example, the electrical and the thermal power balances are still correlated because of the heat pump. In particular, the error in the temperature forecast causes an erroneous computation for the efficiency of the device, that would require, therefore, a different amount of electrical power in order to satisfy the thermal request. This further motivates the adoption of a global scheduler for the microgrid devices such as the one 
proposed in this paper, so as to manage the electrical and the thermal need of the microgrid taking into account any correlation among the systems.

\section{Conclusions}

This paper presents a method for the optimal energy management of a microgrid subject to electric and thermal power requests and provided of a connection to the utility grid. In particular, the algorithm we introduced allows to define the optimal energy exchange profile with the network in a day-ahead scenario. Furthermore, taking into account the possibility to obtain more reliable informations on the forecasted data throughout the day, the method we presented allows to revise such plan in the effort to maintain the network exchange as close as possible to the one agreed with the network manager.

Even if the introduction of a lower-level controller - similar to the one proposed in [5] - is most likely to be needed to compensate for higher-frequency fluctuactions, the effect of an update policy is clearly beneficial to this purpose and it allows for a better coordination of the devices composing the microgrid.

The results of the simulation study show the potentialities of the approach and encourage further research on the topic.

\section{Acknowledgement}

This work has been financed by the Research Fund for the Italian Electrical System under the Contract Agreement between RSE S.p.A. and the Ministry of Economic Development - General Directorate for Nuclear Energy, Renewable Energy and Energy Efficiency - in compliance with the Decree of March 8, 2006.

\section{References}

[1] US Department of Energy, "The smart grid: an introduction", Technical Report, 2009.

[2] R. Lasseter and P. Paigi, "Microgrid: a conceptual solution", IEEE $35^{\text {th }}$ Annual Power Electronics Specialists Conf., vol. 6, 2004, pp. 4285-4290.

[3] A. Parisio, E. Rikos and L. Glielmo, "A model predictive control approach to microgrid operation optimization", IEEE Trans. on Control Systems Technology, vol. 22, no. 5, 2014, pp. 1813-1827.

[4] S. Bracco, F. Delfino, F. Pampararo, M. Robba and M. Rossi, "A mathematical model for the optimal operation of the University of Genoa Smart Polygeneration Microgrid. Evaluation of technical, economic and environmental performance indicators", Energy, vol. 64, 2014, pp. 912922.

[5] S. Raimondi Cominesi, M. Farina, L. Giulioni, B. Picasso and R. Scattolini, "Two-layer predictive control of a microgrid including stochastic energy sources", American Control Conference (ACC), 2015, pp. 918-923.

[6] RSE S.p.A., "La pompa di calore per un comfort sostenibile", Editrice Aikes, 2013.

[7] A. Bemporad and M. Morari, "Control of systems integrating logic, dynamics and constraints", Automatica, vol. 35(3), 1999, pp. 407-427.

[8] F. Torrisi and A. Bemporad, "HYSDEL - A tool for generating computational hybrid models for analysis and synthesis problems", IEEE Trans. on Control Systems Technology, vol. 12, 2004, pp. 235-249. 